\title{
A clinical pathway reduced use of nebulised $\beta$ agonists and length of hospital stay in children with asthma exacerbations
}

\author{
Johnson KB, Blaisdell CJ, Walker A, et al. Effectiveness of a clinical pathway for inpatient asthma management. Pediatrics \\ 2000 Nov;106:1006-12. \\ QUESTION: In children admitted to hospital with asthma exacerbations, does use of a \\ clinical pathway decrease use of nebulised $\beta$ agonists, length of hospital stay, and \\ unplanned health encounters?
}

Design

Randomised \{allocation concealed\}*, blinded (outcome assessors for selected variables), controlled trial.

Setting

An urban, academic medical centre in Baltimore, Maryland, USA.

\section{Patients}

112 children who were $2-18$ years of age, were admitted to hospital with a primary diagnosis of asthma exacerbation, and who were not under the care of an asthma specialist. Exclusion criteria were admission to the intensive care unit and previous enrolment in the study. $98 \%$ of patients (mean age 7.4 y, $64 \%$ boys, $95 \%$ black) were included in the analysis.

\section{Intervention}

55 patients were allocated to a ward with care based on a clinical pathway, which comprised a nurse driven protocol for weaning bronchodilators, regular peak flow measurement, asthma teaching beginning on the day of admission, prescriptions for home treatments provided before discharge, and early coordination of care between the attending physician and private medical doctor. 57 patients were allocated to a ward providing usual care (vital signs taken before $\beta$ agonist administration, inhaler and spacer education, and coordination of post-discharge care).

\section{Main outcome measures}

Number of nebulisation treatments during hospital stay, duration of hospital stay, hospital charges per patient, and number of unplanned urgent care, emergency department, or inpatient encounters for worsening of symptoms within 2 weeks of discharge.

part the Johns Hopkins

Miracle Telethon Funds.

For correspondence:

Dr K B Johnsom

Division of General

Pediatrics, The Johns

Hopkins University

School of Medicine,

CMSC 140, 600 N

Wolfe Street, Baltimore,

MD 21287-3144, USA.

Fax +14105025440 .

\section{Main results}

Patients managed with the clinical pathway had fewer nebulisation treatments $(4.5 \vee 6.5$ doses every $2 \mathrm{~h}$, $\mathrm{p}=0.02 ; 3.7 v 5.9$ doses every $3 \mathrm{~h}, \mathrm{p}=0.002$ ) and shorter hospital stays than those who received usual care (40.3 v $53.7 \mathrm{~h}, \mathrm{p}<0.01$ ), and more were discharged within 24 hours of admission (table). Patients in the clinical pathway group had lower routine (room) charges (US\$2407 v \$3116, $\mathrm{p}<0.001)$ and lower treatment

Clinical pathway based care $v$ usual care for children admitted with asthma exacerbationst

\begin{tabular}{lllll} 
Outcome & $\begin{array}{l}\text { Clinical } \\
\text { pathway }\end{array}$ & $\begin{array}{l}\text { Usual } \\
\text { care }\end{array}$ & RBI $(95 \%$ Cl) & NNT (Cl) \\
$\begin{array}{l}\text { Discharge within } 24 \\
\text { hours of admission }\end{array}$ & $38 \%$ & $15 \%$ & $162 \%(31$ to 442$)$ & 5 (3 to 14) \\
\hline
\end{tabular}

charges (respiratory therapy) $(\$ 42 v \$ 250, \mathrm{p}<0.001)$ than the usual care group, but did not differ for medication or laboratory charges. The groups did not differ for unplanned healthcare encounters.

\section{Conclusion}

In children admitted to hospital with asthma exacerbations, care based on a clinical pathway with a nurse driven weaning protocol decreased use of nebulised $\beta$ agonists and length of hospital stay.

*Information provided by author.

\section{COMMENTARY}

The results of the study by Johnson et al are similar to those of other studies showing that standard asthma treatment protocols lower costs. ${ }^{12}$ Johnson et al's clinical pathway involved an innovative nurse driven weaning protocol. The sample was sufficiently large to detect significant differences between groups. The intervention was provided by nursing staff, who assessed patients before and after each nebulised medication using a standard treatment guideline, and notified the house officer on call, who then determined whether treatment could be weaned.

Strengths of the study include blinding of outcome assessors and inclusion of cost data, which are useful for decision making. Most participants were black, and therefore the generalisibility to other races is unclear. The authors acknowledged that increased resident knowledge during the study and a more favourable nursing ratio in the intervention unit might confound the findings. Therefore, the results should be viewed within these contexts.

The results are relevant to clinicians in paediatric settings that strive to improve cost effectiveness. The study showed that nurses could safely and reliably assess patients, and improve the care process by weaning the frequency of their medication in a timely manner.

The nurse driven clinical pathway is an innovative concept that could be incorporated into work practices. Its successful adoption will depend on the cooperation of physicians and its implementation as a policy framework within the institution. Staff education in respiratory assessment and support from management are also paramount for the successful implementation of a nurse driven asthma protocol.

Cecilia Lau, RN, PhD Adjunct Associate Professor University of Western Sydney, Nepean Currently seconded as Senior Policy Analyst Office of the Chief Nursing Officer NSW Health Department New South Wales, Australia

1 Greineder DK, Loane KC, Parks P. J Allergy Clin Immunol 1999; 103:436-40.

2 Evans R 3rd, LeBailly S, Gordon KK, et al. Chest 1999;116:210S-16S. 